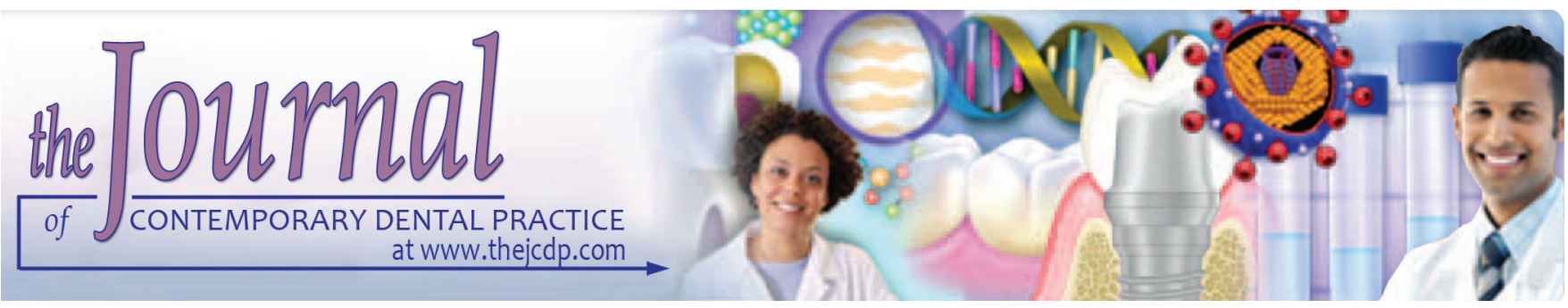

\title{
Evaluation of Hemoglobin Concentration and Hematocrit Values in Temporomandibular Joint Ankylosis Patients in Comparison to Nonankylosed Patients
}

\author{
1Deepinder Singh, ${ }^{2}$ Guneet Kaur, ${ }^{3}$ Dipanshu Bakshi, ${ }^{4}$ Jasjit Sahota, ${ }^{5}$ Ambika Thakur, ${ }^{6}$ Shekhar Grover
}

\begin{abstract}
Aim: The aim of this study is to find if there is any correlation between the hematological parameters and temporomandibular joint (TMJ) ankylosis and severity of the disease in such patients when compared with the nonankylosed patients.
\end{abstract}

Materials and methods: A total of 70 patients with age ranging from 10 to 40 years were included in the study after excluding the subjects according to the inclusion criteria. We categorized the subjects into two major groups: group I: control (nonankylosed/ healthy subjects) and group II: study group (ankylosed subjects) with each group containing 35 subjects $(n=35)$ respectively. A detailed personal and medical history was obtained. The pharynx diameter was also recorded for each patient, and blood investigations using venous blood were done, which included hemoglobin concentration and hematocrit values.

Results: The results of study population showed a mean age of $22 \pm 2.2$ years. The most common etiology reported was trauma $(65.7 \%)$ followed by infections, in which Noma was the most common one $(80 \%)$. The difference of the mean values for hemoglobin and hematocrit concentration, between both the groups, was found to be statistically significant $(p<0.0001)$. Furthermore, a positive correlation was observed between the hemoglobin concentration and duration of ankylosis.

\footnotetext{
${ }^{1}$ Private Practitioner, Department of Oral and Maxillofacial Surgery, Ludhiana, Punjab, India

${ }^{2}$ Private Practitioner, Department of Periodontology, Ludhiana, Punjab, India

${ }^{3,5}$ Department of Dentistry, Chintpurni Medical College \& Hospital, Pathankot, Punjab, India

${ }^{4}$ Department of Periodontics, Institute of Dental Sciences Jammu, Jammu and Kashmir, India

${ }^{6}$ Department of Public Health Dentistry, Maulana Azad Institute of Dental Sciences, New Delhi, India

Corresponding Author: Shekhar Grover, Department of Public Health Dentistry, Maulana Azad Institute of Dental Sciences, New Delhi, India, Phone: +919501544877, e-mail: drshekhargrover@yahoo.co.in
}

Conclusion: This study was an attempt to find a relation between the hemoglobin and hematocrit values in TMJ ankylosis patients so that the clinical treatment and management of such patients during surgeries be improved and may be beneficial for the patient.

Clinical significance: Temporomandibular joint ankylosis patients have to undergo complex surgical treatment, where the risk of excessive blood loss is high. Therefore, considering the complications of blood transfusions, such as infections and other risk factors, these patients can be good subjects for autologous blood transfusions, which help in improvement of the overall well-being of the patient.

Keywords: Airway obstruction, Hematocrit, Hemoglobin, Temporomandibular joint ankylosis.

How to cite this article: Singh D, Kaur G, Bakshi D, Sahota J, Thakur A, Grover S. Evaluation of Hemoglobin Concentration and Hematocrit Values in Temporomandibular Joint Ankylosis Patients in Comparison to Nonankylosed Patients. J Contemp Dent Pract 2018;19(2):210-213.

Source of support: Nil

Conflict of interest: None

\section{INTRODUCTION}

The TMJ is a highly specialized joint, which has the articulating surfaces of bones and is covered by an avascular tissue rather than hyaline cartilage. Any trauma or infection to this tissue can lead to fibrosis of the soft tissue and further develop into a pathological condition in which bony union occurs, which is termed as bony ankylosis. This deformity can be unilateral or bilateral, and its severity is related to both age and duration. ${ }^{1}$ The most common cause of ankylosis according to some studies is said to be trauma. ${ }^{2}$

If this deformity occurs in a growing age, as trauma is the most common in young patients, then there is a shortening of mandibular rami and decrease in the space 
between the mandibular angles, which finally results in a narrow oropharyngeal airway. Along with this, there is a decrease in tonicity of oropharyngeal muscles, which can lead to inability in opening mouth. Due to all such changes, the airway resistance increases and upper airway obstruction occurs. ${ }^{3,4}$

As we know when airway obstruction occurs, it leads to decreased oxygen perfusion to the tissues. However, in some physiological conditions like in high altitude areas, there is a physiological adaptation of the body to this decreased oxygen supply by an increase in oxygencarrying capacity of the red blood cells (RBCs), increase in RBC count or increased hematocrit values. ${ }^{5}$

Very few studies have been published in the literature that emphasized on the airway obstruction and the importance of hematological and hematocrit values in such patients. ${ }^{6,7}$ Thus, this study aimed to find if there is any correlation between the hematological parameters and TMJ ankylosis and severity of the disease. Also, the study aims to provide some significant data regarding this so that it can be helpful to the patients in terms of treatment and management.

\section{MATERIALS AND METHODS}

The present study included all the patients with TMJ ankylosis who reported to the department. An informed consent was obtained before commencement of the study from the patients who were included in this study. Ethical clearance was obtained from the Institutional Ethical Committee before the commencement of the study.

Inclusion criteria included all the patients who presented with both clinical and radiographic presentation of TMJ ankylosis. Even children with diagnosed case of ankylosis were included in the study. Those subjects who had heart diseases, chronic obstructive airway diseases, history of smoking, and hemoglobinopathies were excluded from the study.

A total of 70 patients with age ranging from 10 to 40 years were included in the study after excluding the subjects according to the inclusion criteria. We categorized the subjects into two major groups: group I: control (nonankylosed/healthy subjects) and group II: study group (ankylosed subjects), with each group containing 35 subjects $(n=35)$ respectively. The nonankylosed group included healthy individuals with age and gender following matching the study group.

A detailed personal and medical history was obtained from the subjects included in the study. The desired information extracted from the history included sex, age, type of ankylosis, unilateral/bilateral, etiology behind it, and duration. On examination, mouth opening was recorded with the help of a ruler by measuring the interincisal distance between the incisal edges of maxillary and mandibular central incisors.

The pharynx diameter was also recorded for each patient by measuring the distance between one mandibular angle stretching across the upper part of the neck anteriorly and the contralateral angle when the neck is in an extended position.

For the blood investigations, venous blood was withdrawn from each patient and sent to the laboratory for obtaining hemoglobin concentration and hematocrit values. All the data obtained were recorded and tabulated and sent for statistical analysis using Statistical Package for the Social Sciences (version 13, SPSS Inc., Chicago Illinois). Chi-square test was applied for the $p$-values, and $p \leq 0.05$ was considered to be statistically significant. Multivariate analysis was done for intergroup comparison.

\section{RESULTS}

In the present study, a total of 70 subjects were included, which were further categorized into ankylosed and nonankylosed groups ( $\mathrm{n}=35$ in each group). The ankylosed subjects (study group, group II) comprised patients with a mean age of $22 \pm 2.1$ years, and the mean duration of presentation of TMJ ankylosis was 12 years. In this study, not much of gender difference was observed, with males forming $48.5 \%$ and females forming $51.4 \%$ of the study population. The most common etiology reported by the patients for TMJ ankylosis came to be trauma (65.7\%) followed by infections, in which Noma was the most common one (80\%) (Table 1 and Graph 1 ).

For the hemoglobin and hematocrit concentration, the mean value observed for group I was $11.3 \pm 0.52$ and $32.8 \pm 2.54$ respectively. Similarly, for group II, it was $12.1 \pm 0.82$ and $38.6 \pm 1.42$ respectively (Table 2 and Graph 2). The difference between both the groups was found to be statistically significant $(p<0.0001)$.

A positive correlation was observed between the hemoglobin concentration and duration of ankylosis

Table 1: Mean values of the clinical parameters of the study group (group II)

\begin{tabular}{ll}
\hline Clinical parameter & Mean $\pm S D(n=35)$ \\
\hline Age & $22 \pm 2.1$ \\
Sex & \\
Male & $48.5(\mathrm{n}=17)$ \\
Female & $51.4(\mathrm{n}=18)$ \\
Etiology & \\
Trauma & $65.7(\mathrm{n}=23)$ \\
Infections (total) & $28.5(\mathrm{n}=10)$ \\
Ear infection & $25(\mathrm{n}=2)$ \\
Noma (cancrum oris) & $80(\mathrm{n}=8)$ \\
Unknown & $5.7(\mathrm{n}=2)$ \\
Duration (years) & 12 \\
\hline
\end{tabular}

SD: Standard deviation 


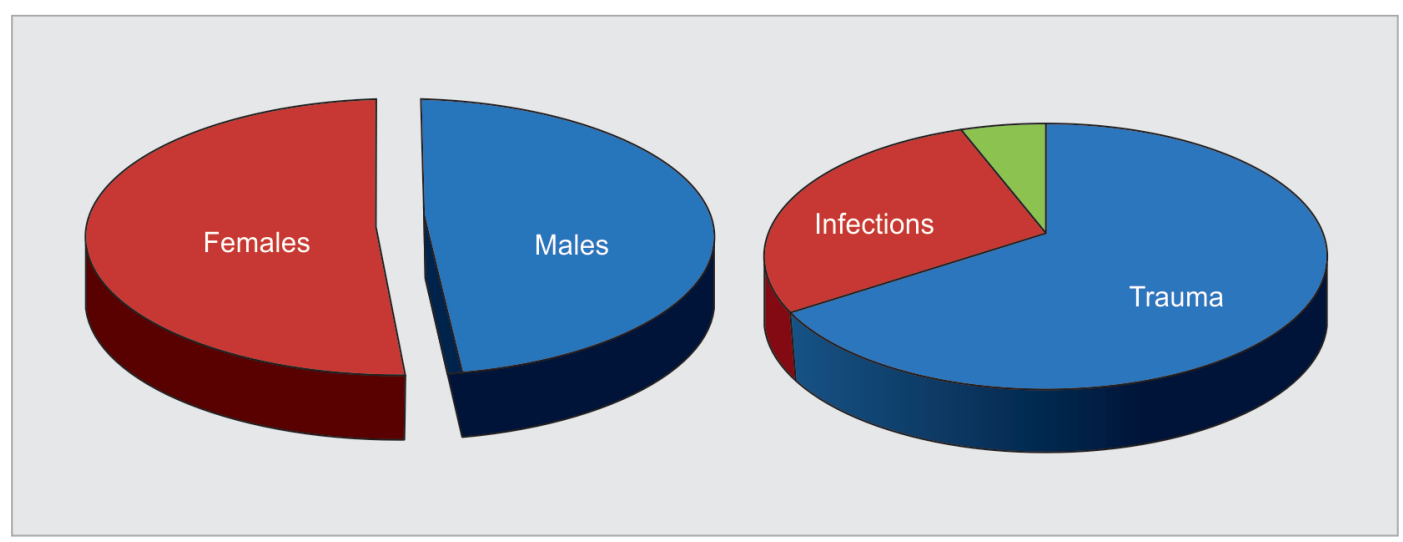

Graph 1: Means of clinical parameter among study groups

Table 2: Comparison of hemoglobin and hematocrit values of both control and study groups

\begin{tabular}{lllr}
\hline Variables & $\begin{array}{l}\text { Group I } \\
\text { (nonankylosed) }\end{array}$ & $\begin{array}{l}\text { Group II } \\
\text { (ankylosed) }\end{array}$ & p-value \\
\hline Hemoglobin $(\mathrm{Hb} \%)$ & $11.3 \pm 0.52$ & $12.1 \pm 0.82$ & $<0.0001$ \\
Hematocrit $(\%)$ & $32.8 \pm 2.54$ & $38.6 \pm 1.42$ & $<0.0001$ \\
\hline
\end{tabular}

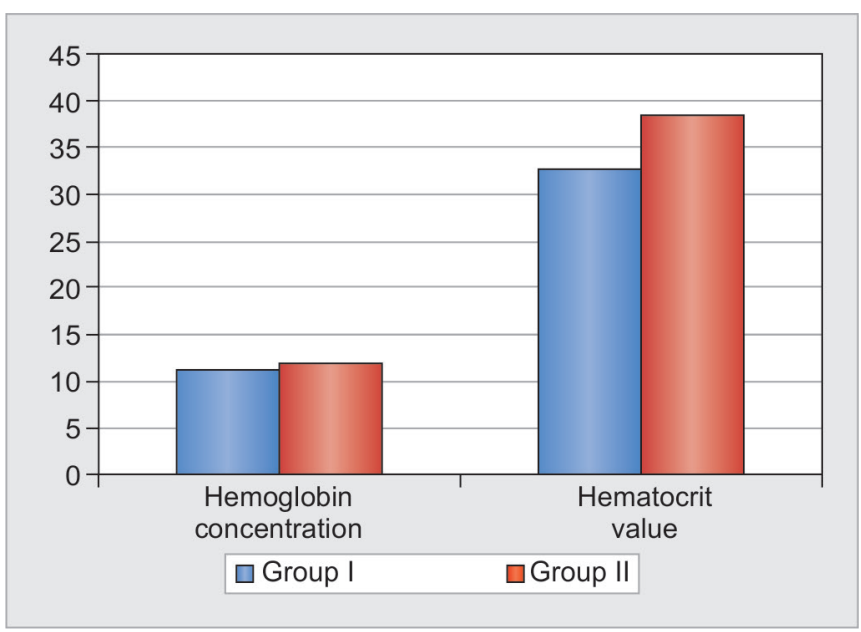

Graph 2: Comparison of hemoglobin and hematocrit values of both control and study groups

$(\mathrm{r}=0.164, \mathrm{p}=0.032)$ and hematocrit value $(\mathrm{r}=0.492$, $p=0.038$ ) respectively. Furthermore, when a multivariate analysis was done, it showed that age and gender had no effect on the values of hematocrit and hemoglobin concentration when both the groups were compared.

\section{DISCUSSION}

Temporomandibular joint ankylosis is a pathological condition which not only leads to decrease in tonicity of oropharyngeal muscles but also inability in opening mouth. Thus, the airway resistance increases and upper airway obstruction occurs, which leads to a state of relative hypoxia. This condition clinically may be reported by patients with TMJ ankylosis as difficulty in breathing, which is related to the above-mentioned findings. It can be hypothesized in the light of above relationship that hematological parameters of such patients will definitely be affected. Therefore, hypothesizing this relationship, this study aimed to find a correlation between the hematological parameters and TMJ ankylosis among ankylosed and nonankylosed patients.

The study design for this study included a total sample of 70 subjects divided into two groups, one with ankylosed patients $(n=35)$ and the other one included nonankylosed subjects $(n=35)$ with comparable age and gender. In our study, the mean age group of the ankylosed group of patients was observed to be $22 \pm 2.1$ years, and the mean duration of presentation of the problem of TMJ ankylosis was reported to be 12 years.

The results of the present study firstly showed no significant gender difference in the sample undertaken for our study as males and females were found to be almost equally divided with the female population being slightly more than the males. When etiology was considered, trauma was the most common cause for ankylosis, as the maximum number of cases reported in our study gave a positive history of trauma. Infections, such as Noma and ear infections were found to be less common causes in this study. Few patients did not have any relevant history regarding the etiology, and thus were categorized under unknown category. Güven ${ }^{6}$ in his study also revealed a predominance of traumatic etiology, with the highest incidence between the ages of 11 and 20 years.

As this study mainly aimed to find the difference between the hemoglobin and hematocrit levels between patients with TMJ ankylosis and the patients without it, it was observed that both hemoglobin percentage and hematocrit levels were found to be on the higher side in the ankylosed group, as compared with the nonankylosed group, and this difference was found to be statistically significant for both $(\mathrm{p}<0.0001)$. Our results were in accordance with Osunde et al, ${ }^{7}$ who also gave similar results in their study. As proposed, the reason behind this could be the body's compensatory mechanisms to 
deliver sufficient oxygen to the tissues when it is compromised. It is already proven and documented in the previous studies that due to ankylosis, defects, such as retrognathia, relative macroglossia, and alteration of the normal anatomy of the upper airway can cause airway obstruction in such patients. ${ }^{8-10}$ Thus, altogether, the oropharyngeal airway is affected, which can lead to decreased RBCs and oxygen perfusion to the tissue, thus creating a hypoxic condition. ${ }^{11-13}$

To prevent hypoxia in the tissues, the body responds to counteract this by an increase in blood levels of the RBCs with the help of excess production of a glycoprotein hormone erythropoietin (EPO) by the body. Various rat model studies have reported that these so-called "oxygen sensors" are located on the kidneys and regulate the production of EPO hormone by the kidneys in such hypoxic conditions. The molecular basis of this mechanism is proposed as: First, hypoxia in the kidneys can lead to increased production of metabolic mediators, like prostaglandins, which conversely stimulate EPO gene transcription by increasing cellular levels of second messenger molecules. Second, some receptors like heme protein control EPO production by an oxygen-dependent conformational change, which could mediate the signal transduction process. ${ }^{14,15}$ This EPO hormone secretion has the main role is increasing the systemic oxygen-carrying capacity. It even increases the RBC cell concentrations by stimulating the formation and differentiation of erythroid precursor cells in the bone marrow. ${ }^{14}$

In our study, a positive correlation was also observed between both hemoglobin concentration and duration of ankylosis in the patients, thus proving that the longer the history of ankylosis more chances are that the patient will have a good hemoglobin and hematocrit concentration. Again in our study, age and gender have no correlation with the hemoglobin and hematocrit concentration.

The results of our study show that ankylosed patients have good hemoglobin and hematocrit values as compared with the nonankylosed group. If we consider the clinical significance of this study, we can say that the hematological values are important for consideration when treatment of such patients is planned. Such patients have to undergo complex surgical treatments which can be one or more. The major problems faced during such surgeries are the risk of excessive blood loss. Therefore, considering the complications of blood transfusions, such as infections and other risk factors, these patients can be good subjects for autologous blood transfusions, which can be beneficial for the patient and help in improvement of the overall well-being of the patient.

\section{CONCLUSION}

This study was an attempt to find the relation between the hemoglobin and hematocrit values in TMJ ankylosis patients. However, still more studies are required to validate our results with more sample size so that the clinical treatment and management of such patients during surgeries may be improved and may be beneficial for the patient.

\section{REFERENCES}

1. Wahal R. Temporo-mandibular joint ankylosis—-the difficult airway. J Oral Biol Craniofac Res 2015 May-Aug;5(2):57-58.

2. Gupta VK, Mehrotra D, Malhotra S, Kumar S, Agarwal GG, Pal US. An epidemiological study of temporomandibular joint ankylosis. Natl J Maxillofac Surg 2012 Jan-Jul;3(1):25-30.

3. Zanaty O, El Metainy S, Abo Alia D, Medra A. Improvement in the airway after mandibular distraction osteogenesis surgery in children with temporomandibular joint ankylosis and mandibular hypoplasia. Paediatr Anaesth 2016 Apr;26(4):399-404.

4. Shi Z, Guo C, Awad M. Hyaluronate for temporomandibular joint disorders. Cochrane Database Syst Rev 2003;1:CD002970.

5. Storz JF. Evolution. Genes for high altitudes. Science 2010 Jul;329(5987):40-41.

6. Güven O. A clinical study on temporomandibular joint ankylosis. Auris Nasus Larynx 2000 Jan;27(1):27-33.

7. Osunde DO, Akhiwu BI, Omeje KU, Amole OI, Efunkoya AA. Correlation between hemoglobin concentration and hematocrit values in patients with temporomandibular joint ankylosis and nonankylosed patients. J Health Res Rev 2017 Oct;4(3):137-142.

8. Prabhakar AR, Rai KK, Bedi S. Management of congenital bilateral temporomandibular joint ankylosis with secondary mandibular hypoplasia. J Pediatr Surg 2008 Oct;43(10):e27-e30.

9. Felstead AM, Revington PJ. Surgical management of temporomandibular joint ankylosis in ankylosing spondylitis. Int J Rheumatol 2011 Mar;2011:854167.

10. Alday LE, Vega PJ, Heller A. Congenital ankylosis of the temporomandibular joint: resultant upper airway obstruction and cor pulmonale. Chest 1979 Mar;75(3):384-386.

11. Zhong R, Liu H, Wang H, Li X, He Z, Gangla M, Zhang J, Han D, Liu J. Adaption to high altitude: an evaluation of the storage quality of suspended red blood cells prepared from the whole blood of Tibetan plateau migrants. PLoS One 2015 Dec;10(12):e0144201.

12. Blanchard KL, Acquaviva AM, Galson DL, Bunn HF. Hypoxic induction of the human erythropoietin gene: cooperation between the promoter and enhancer, each of which contains steroid receptor response elements. Mol Cell Biol 1992 Dec;12(12):5373-5385.

13. Braimah RO, Oladejo T, Olarinoye TO, Adetoye AO, Osho PO. A multidisciplinary approach to the management of temporomandibular joint ankylosis in a sickle-cell anemia patient in a resource-limited setting. Ann Maxillofac Surg 2016 Jan-Jun;6(1):130-134.

14. Scholz H, Schurek HJ, Eckardt KU, Bauer C. Role of erythropoietin in adaptation to hypoxia. Experientia 1990 Dec;46(11-12):1197-1201.

15. Fisher JW. Erythropoietin: physiology and pharmacology update. Exp Biol Med (Maywood) 2003 Jan;228(1):1-4. 